\title{
Is Pedicle Screw Dynamic Stabilisation a Possible Management of Lumbar Diseases with A Low-Grade Instability?
}

\author{
R Ciarpaglini MD $^{1}$ and Danilo Esaltato ${ }^{2 *}$ \\ ${ }^{1}$ Neurosurgical Department, Lindenhofspital Bern, Switzerland \\ ${ }^{2}$ Faculty of Human Medicine, University of Bern, Switzerland
}

*Corresponding author: Danilo Esaltato, Faculty of Human Medicine, University of Bern, Hochschulstrasse 6, 3012 Bern, Switzerland.

To Cite This Article: R Ciarpaglini MD, Danilo Esaltato. Is Pedicle Screw Dynamic Stabilisation a Possible Management of Lumbar Diseases with A Low-Grade Instability?. Am J Biomed Sci \& Res. 2021 - 12(1). AJBSR.MS.ID.001707. DOI: 10.34297/AJBSR.2021.12.001707.

Received: 䟧 January 25, 2021; Published: 眥 February 16, 2021

\begin{abstract}
Background: A dynamic stabilisation of the lumbar spine can be performed in selected cases of degenerative disc diseases as an alternative to the established but more invasive rigid fusion surgery. The purpose of our study was to assess the clinical and radiological results of dynamic stabilisation of the lumbar spine with pedicle screws and polyetheretherketone (PEEK) rods.

Methods: Between March 2018 and March 2020, forty-two patients underwent forty-three consecutive operations for a dynamic lumbar stabilisation. Pre- and postoperative radiological assessment, operative reports and pre- and postoperative clinical documents were analysed retrospectively.

Results: The clinical assessment showed on average a postoperative reduction of the back pain from 5.2 to 3.1 (visual analogue scale, 0 to 10 ) and of the leg pain from 6.4 to 1.8 (visual analogue scale, 0 to 10). The radiological controls provided stable results except in one asymptomatic case where a possible mobilisation of a pedicle screw was observed. Three patients (7\%) needed a reoperation and in three patients (7\%) intraoperative complications have been observed.

Conclusion: With the retrospective analysis of the clinical and radiological results of a population of 42 patients (43 operations) consecutively treated with a dynamic lumbar stabilisation surgery the safety of the system can be confirmed. No significant radiological complications have been observed. The pain level of back and leg pain decreased after the operation and remained stable in the controls.
\end{abstract}

Keywords: Degenerative spine disease; lumbar spine; dynamic stabilization; pedicle screws; PEEK rods

Abbreviations: PEEK: Polyetheretherketone; VAS: Visual analogue scale

\section{Introduction}

Degenerative diseases of the lumbar spine can cause different degrees of instability. The treatment of these pathologies often needs a decompression and, in some cases, a stabilisation of the involved segments. Rigid spinal fusion is considered the gold standard to treat degenerative diseases of the lumbar spine associated with instability. The increased load on the adjacent segment with a risk of developing a segmental instability and stenosis is however a well-known long-term side effect of rigid fusion surgery. Sometimes the instability associated with the degenerative pathology is not so severe to require a rigid stabilisation including postero-lateral pedicle screwing and interbody fusion. Especially in elderly patients, a dynamic stabilising system can be considered to minimize the surgical risks reducing operating time and blood loss. Dynamic stabilisation avoids moreover to perform an extensive discectomy reducing the risks of interbody cages' subsidence and other complications associated with poor bone quality. The dynamic stabilisation can provide an acceleration of the degenerative cascade according to [1] tending to a spontaneous 
fusion of the spine in a more progressive way compared to a rigid fusion. This delayed fusion could be achieved through a facet joints ankylosis and a progressive dehydration of the intervertebral discs.

\section{Objective}

The aim of this study is to assess the clinical results of dynamic stabilisation of the lumbar spine with Viper SC and Polyetheretherketone (PEEK) rods. The retrospective analysis of the radiological control studies in a cohort of forty-two patients provides also information concerning the stability of the segments before and after the operation. The safety of the procedure is, besides, evaluated through the analysis of the perioperative and postoperative complication rate.

\section{Methods}

Between March 2018 and March 2020, forty-two patients underwent forty-three consecutive operations for a dynamic stabilisation using Viper SC and PEEK rods. The surgical procedures were performed by the same spinal surgeon in the Linden hospital neurosurgical Unit in Bern (Switzerland). All the patients treated with this system during this period were included. Patients with spondylolisthesis grade II-IV, pathologic fractures, active infections, or spinal metastasis were not suitable for treatment with Viper SC. Pre- and postoperative radiological assessment, operative reports and pre- and postoperative clinical documents were analysed retrospectively. The clinical assessment was performed using the Visual Analogue Scale (VAS) for back and leg pain. A complete neurological examination was performed before and after the surgical procedure and the results are reported in this clinical study. The preoperative radiological assessment always included an MRI of the lumbar spine. Functional X-Rays of the lumbar spine were performed every time a segmental instability was suspected. In some patients a standing X-Ray of the entire spine and a CT were also performed. Control X-Rays were performed 2, 6 and 12 months after surgery. Further radiological investigations were performed just in selected indications. All participants provided written informed consent.

\section{Results}

The population consisted of 42 patients consecutively operated during a period of 2 years by the same spinal surgeon in the same Hospital. A total number of 43 surgical procedures using Viper SC were performed as one patient underwent two operations at two different levels. A preoperative pain syndrome was present at least during 3 months before surgery for most of the patient population and all possible alternative treatment (physical treatment, steroids injections) were ineffective to control the pain syndrome. In 4 cases an abrupt beginning of the symptoms was observed and required an urgent surgical treatment. Every patient was under treatment with anti-inflammatory and antalgic drugs. The preoperative functional X-Rays were performed by suspicion of segmental instability on 29 cases (67\%). They showed a light instability in 17 patients (increase of vertebral slip until $3 \mathrm{~mm}$ ). In eight patients the vertebral slip was higher. According to the Meyerding classification of degenerative Spondylolisthesis six patients presented a grade I anterolisthesis. No patient of the analysed population presented a higher level of anterolisthesis (Table 1 ).

Table 1: Demographic and preoperative characteristics of the patients.

\begin{tabular}{|c|c|}
\hline No. of patients & 42 \\
\hline No. of operations & 43 \\
\hline Female/Male & $25 / 17$ \\
\hline Mean age [years] & $69(\mathrm{SD} \pm 9)$ \\
\hline $\begin{array}{l}\text { No. of patients with previous } \\
\text { spinal operations }\end{array}$ & $17(40 \%)$ \\
\hline \multicolumn{2}{|c|}{ Primary indication for surgery } \\
\hline Spinal canal stenosis & $22(51 \%)$ \\
\hline Disc herniation & $8(19 \%)$ \\
\hline Antherolisthesis & $6(14 \%)$ \\
\hline Foraminal stenosis & $4(9 \%)$ \\
\hline Retrolisthesis & $3(7 \%)$ \\
\hline Preoperative neurological deficit & $12(28 \%)$ \\
\hline
\end{tabular}

\section{SD: Standard Deviation}

All the patients were treated with the combination of Viper SC and PEEK rods. In one patient a three-level surgery was performed (L2-L5), six patients were treated at two levels (4 times L3-L5 and 2 times L4-S1) and in 36 cases (84\%). a dynamic mono-segmental stabilisation was realized. The most treated level was L4/L5 (34 cases, 79\%). L3/L4 was treated in 9 cases, L5/S1 in 5 cases and L2/L3 in 3 cases. The level of decompression matched the level of stabilisation in 31 cases (72\%). In seven cases the decompression concerned also adjacent non-stabilised segments and in five cases not all the stabilised segments needed direct microsurgical decompression. Just two patients required a pure percutaneous stabilisation. All the operations, with exception of the two percutaneous procedures, were performed using a microsurgical technique and intraoperative C-Arm radiological control. In one case an 0-Arm navigation system was used. The average surgical time was 2 hours and 13 minutes and the blood loss was on average $164 \mathrm{ml} /$ case. Intraoperative complications were observed in three cases (7\%): two patients with CSF-leak and one patient with medial position of a pedicle screw. Among them, just one patient required a surgical revision: The Viper SC screw was in this patient repositioned.

In no case the association of a supplementary stabilising device was necessary. A posterolateral bone graft was used in 30 cases (70\%). In 16 cases (37\%) Demineralized Bone Matrix (DBX) was used and in 14 cases (33\%) autologous bone. The median duration 
of the hospitalisation was eight days ( $\min 6, \max 13)$. Three patients required a reoperation: one patient because of the malposition of a pedicle screw three days after surgery; the other patient developed a low-grade infection and required a revision five weeks after the first operation; and in one patient the dynamic stabilisation was changed with a rigid system five months later because of persisting lumbar and radicular pain. In the case with pedicular screw malposition the same Viper SC screw was correctly repositioned during the revision surgery three days after the first surgery. In the case with low grade infection the removal of the semirigid stabilisation system was necessary at the time of the revision surgery: Viper SC screws and PEEK rods were replaced with thicker CFX Viper screws and titan rods. Clinical and radiological checks were performed 2, 6 and 12 months after surgery. The average follow-up time was 12 months
(SD \pm 6.7 months) (Figure 1). The clinical assessment showed a postoperative reduction of the back pain (VAS, 0 to 10) level from 5.2 to 3.1 on average $( \pm 1.2)$. In nine cases $(21 \%)$ the postoperative pain level for the lumbar pain was not reduced and one patient had a light worsening of the preoperative lumbar pain level. Concerning the radicular symptoms, a dramatic postoperative reduction of the leg pain level (VAS, 0 to 10 ) was observed decreasing from 6.4 to $1,8( \pm 2.0)$ on average. A statistically height significant $(p<0,01)$ postoperative reduction of VAS was calculated for both back and leg pain. The radiological checks provided stable radiological results except in one case where a possible mobilisation of a pedicle screw was observed eight weeks after surgery. In this case no supplementary investigation was performed as the patient had no correlated symptoms (Figure 2).
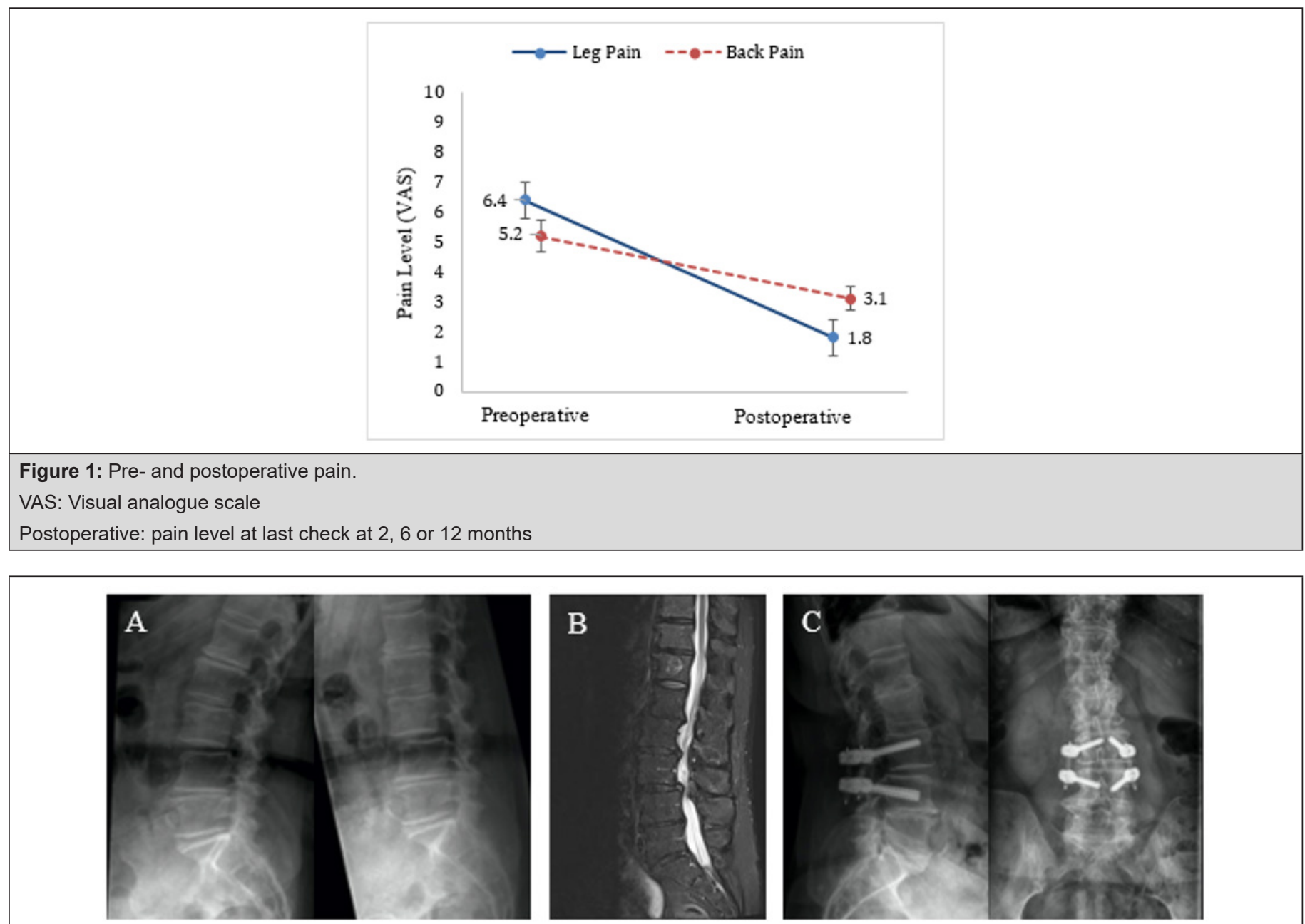

Figure 2: Case example.

Woman, 81 years, preoperative refractory back (VAS 6/10) and leg pain (VAS 6/10),

Surgical procedure: Decompression L2/L3, L3/L4 and dynamic stabilization L3/L4.

Follow-up: 16 months. Postoperative stable radiological results and pain reduction for back (VAS 4/10) and leg (VAS 1/10) pain.

A: Preoperative functional radiography showing segmental instability L3/L4

(3 $\mathrm{mm}$, Antherolisthesis Meyerding grade I)

B: Preoperative MRI showing spinal stenosis L2/L3 and L3/L4

C: Postoperative radiography. 


\section{Discussion}

Even if rigid fusion is considered the gold standard treatment for degenerative lumbar instabilities, the results of several long-term follow-up studies have suggested that it might cause deterioration of the adjacent segment 2-5. Lehmann et al.6 reviewed 33 patients with a follow-up over 20 years after posterior spinal fusion and reported a 30\% incidence of spinal stenosis on the adjacent level. Park et al. reported in a comparable follow-up period a revision rate of adjacent segment diseases after fusion ranging between 5 and $18 \%$. Many authors $[2,3,5,7,8]$ pointed out that interbody fusion may affect the load distribution on the adjacent discs creating a higher intradiscal pressure. Starting from these assumptions dynamic stabilization systems started to be used in the common practice for degenerative diseases. We learned that a segmental kyphosis and an extensive facectomy are contraindications for this surgical indication predicting an anterior column insufficiency. PEEK rod systems are well-known devices introduced with the aim to achieve a pedicle-based posterolateral stabilisation avoiding increasing the mechanical stress at the adjacent segment of the fusion level $[4,9,10]$. PEEK rod systems were approved by the FDA in 2007 and many clinical studies concerning the use of PEEK for fusion procedures are available in literature [11,12]. PEEK rods have an elastic modulus between that of the cancellous and cortical bone and present full biocompatibility in vivo [13].

Hoppe et al. [14] reported comparable long-term results for monosegmental dynamic and rigid stabilisation for degenerative spondylolisthesis concluding that semi-rigid fusion represents a valid alternative to fusion surgery. Even if some studies reported screw loosening as the commonest complication for pedicle-based dynamic stabilization [15-17] we did not observe any symptomatic case of screw loosening in our series. In one case a light displacement of a screw in the postoperative X Rays checks was not correlated to symptoms. In our series the clinical postoperative results were very positive and the leg pain score decreased after the operation and remained stable in the follow-up. Persistent lumbar pain was observed in nine cases (21\%) but most patients experienced a reduction of the lumbar pain postoperatively confirming that the stabilisation provided an adequate support of the decompressed level. These clinical results are comparable with those of a rigid fusion surgery [14]. The length of the hospitalisation (average eight days) and of the surgical procedure time (133 minutes on average) confirmed the quite uncomplicated intra-and postoperative clinical development even on an elderly population (average age 69 years. $\mathrm{SD} \pm 9$ years).

\section{Conclusion}

Through the retrospective analysis of the clinical and radiological results of a population of 42 patients (43 operations) consecutively treated with Viper SC the safety of the system can be confirmed. Indeed, just three cases required a revision surgery but just in one case the revision was due to an insufficient stabilisation of the segment and a rigid stabilisation with interbody fusion was performed. Even if the mean follow-up is short (mean 12 months, $\mathrm{SD} \pm 6.7$ months), no significant radiological complication such as screw-loosening was observed. Further studies with clinical and radiological long-term follow-up are necessary to support the evidence that dynamic stabilisation may be used in low-grade degenerative instability preventing an adjacent segment pathology. Further research should be performed also to assess which grade of instability can be treated with a dynamic stabilisation and which require a rigid fusion. The individuation of clear criteria would allow a proper patient selection and a successful tailored surgical strategy.

\section{Conflict of Interest}

R. Ciarpaglini MD discloses his scientific clinical research collaboration with De Puy Synthes Spine. For the remaining author, none were declared.

\section{References}

1. Kirkaldy-Willis W, Wedge J, Yong-Hing K, Reilly J (1978) Pathology and pathogenesis of lumbar spondylosis and stenosis. Spine (Phila Pa 1976) 3(4): 319-328.

2. Kanayama M, Togawa D, Hashimoto T (2009) Motion-preserving Surgery Can Prevent Early Breakdown of Adjacent Segments Comparison of Posterior Dynamic Stabilization with Spinal Fusion. J Spinal Disord Tech 22(7): 463-467

3. Kelly MP, Mok JM (2010) Dynamic Constructs for Spinal Fusion: An Evidence-Based Review. Orthop Clin NA 41(2): 203-215.

4. Lee CS, Hwang CJ, Lee S-W, Young-Joon Ahn, Yung-Tae Kim, et al. (2009) Risk factors for adjacent segment disease after lumbar fusion. Eur Spine J 18(11): 1637-1643.

5. Sudo H, Oda I, Abumi K, Manabu Ito, Yoshihisa Kotani, et al. (2003) In vitro biomechanical effects of reconstruction on adjacent motion segment: comparison of aligned/kyphotic posterolateral fusion with aligned posterior lumbar interbody fusion/posterolateral fusion. J Neurosurg 99(Spine 2): 221-228.

6. Lehman T, Spratt K, Tozzi J, J N Weinstein, S J Reinarz, et al. (1987) Longterm Follow-up of Lower Lumbar Fusion Patients. Spine (Phila Pa 1976) 12(2): 97-104.

7. Park P, Garton HJ, Gala VC, Hoff JT, Mcgillicuddy JE (2004) Adjacent Segment Disease after Lumbar or Lumbosacral Fusion: Review of the Literature. Spine (Phila Pa 1976) 29(17): 1938-1944.

8. Wang JC, Arnold PM, Hermsmeyer JT, Norvell DC (2012) Do Lumbar Motion Preserving Devices Reduce the Risk of Adjacent Segment Pathology Compared With Fusion Surgery? Spine J 37(22): 133-143.

9. Colangeli S, Barbanti Brodano G, Gasbarrini A, S Bandiera, A Mesfin, et al. (2015) Polyetheretherketone (PEEK) rods: short-term results in lumbar spine degenerative disease. J Neurosurg Sci 59(2): 91-96.

10. Huang W, Chang Z, Song R, Zhou K, Yu X (2016) Non-fusion procedure using PEEK rod systems for lumbar degenerative diseases: clinical experience with a 2-year follow-up. BMC Musculoskelet Disord 17: 53. 
11. Athanasakopoulos M, Mavrogenis A, Triantafyllopoulos G, Koufos S Pneumaticos S (2013) Posterior Spinal Fusion Using Pedicle Screws. Orthopedics 36(7): e951-957.

12. Qi L, Li M, Zhang S, Xue J, Si H (2013) Comparative effectiveness of PEEK rods versus titanium alloy rods in lumbar fusion : A preliminary report. Acta Neurochir (Wien) 155: 1187-1193.

13. Kurtz SM, Lanman TH, Higgs G (2013) Retrieval analysis of PEEK rods for posterior fusion and motion preservation. Eur Spine J 22: 2752-2759.

14. Hoppe S, Schwarzenbach O, Aghayev E, Bonel H (2016) Long-term Outcome After Monosegmental L4 / 5 Stabilization for Degenerative Spondylolisthesis with the Dynesys Device. Clin Spine Surg 29(2): 72-77.
15. Fritzell P, Hägg O, Wessberg P, Nordwall A (2002) Chronic Low Back Pain and Fusion: A Comparison of Three Surgical Techniques A Prospective Multicenter Randomized Study from the Swedish Lumbar Spine Study Group. Spine (Phila Pa 1976) 27(11): 1131-1141.

16. Sapkas G, Mavrogenis AF, Starantzis KA, Soultanis K, Kokkalis ZT, et al. (2012) Outcome of a Dynamic Neutralization System for the Spine. Orthopedics 35(10): 1497-1502.

17. Stoll TM, Dubois G, Schwarzenbach 0. (2002) The dynamic neutralization system for the spine : a multi-center study of a novel non-fusion system. Eur Spine J 11(Suppl 2): 170-178. 